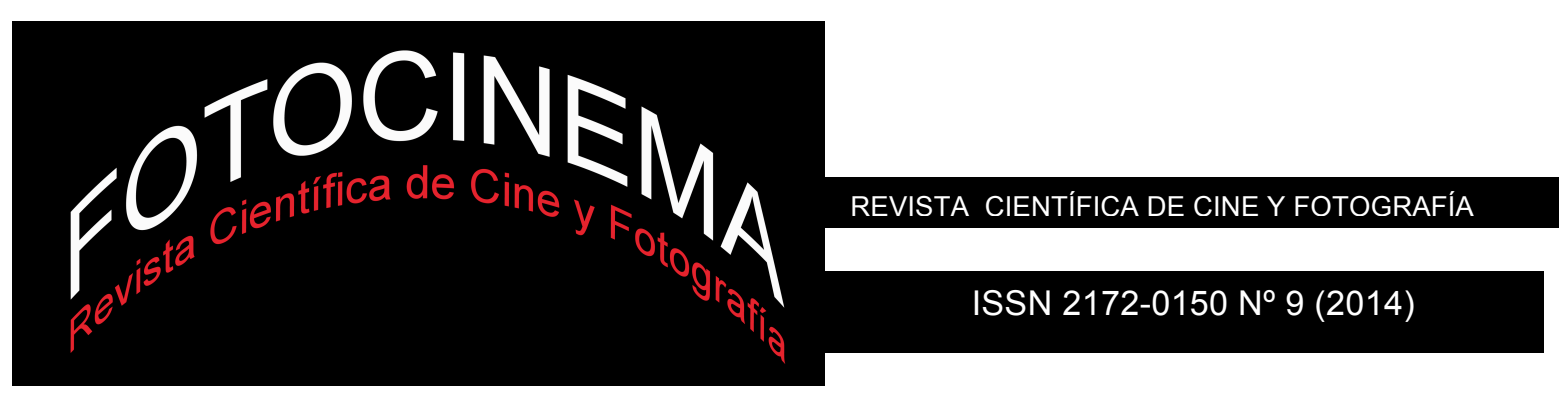

\title{
FOTOCINEMA, LA CONSOLIDACIÓN DE UNA REVISTA DE CINE Y FOTOGRAFÍA
}

\section{Nekane Parejo y Agustín Gómez \\ Directores de FOTOCINEMA}

En este mes, con su número 9, FOTOCINEMA cumple 5 años y queremos celebrarlo con todos vosotros compartiendo algunos datos:

* Estamos representados en más de 26 plataformas de evaluación, bases de datos y catálogos de bibliotecas, y en proceso de entrar en algunas de referencia como Scopus.

* 118 artículos publicados de investigaciones y estudios.

* Los profesores e investigadores proceden de 36 universidades. Excepto Castilla la Mancha, Extremadura y La Rioja están personificadas todas las comunidades autónomas de España. Las mejor representadas son la de Madrid con cinco -Universidad Complutense, Universidad Rey Juan Carlos, Universidad Europea Miguel de Cervantes, Universidad Camilo José Cela y Universidad Carlos III- y Andalucía con otras cinco -Universidad de Córdoba, Universidad de Málaga, Universidad Pablo de Olavide, Universidad de Sevilla y Universidad de Granada-.

* El carácter internacional, que siempre tuvimos presente para esta revista, ha dado como resultado la publicación de artículos en francés, inglés y portugués, y que profesores de 11 universidades extranjeras estén entre nuestros artículos. Llamativo es el que haya seis francesas -Sorbonne Nouvelle Paris 3, Université Diderot-Paris 7, Université Paris 8, Paris X Ouest Nanterre la Défense, Université Rennes 2 y Universidad de Limoges-.

* Si en el número 1 publicamos siete artículos en el actual son catorce. Esto supone un esfuerzo considerable del que nos sentimos orgullosos y del que no 
podemos por menos que agradecer a todos los autores la confianza puesta en nosotros, incluidos aquellos cuyos artículos fueron rechazados, por supuesto a nuestros lectores y especialmente a los evaluadores que realizan un imprescindible trabajo y que hacen que los textos mejoren. En los dos números de 2014 estos han sido 58: Ainara de Miguel, Alfonso Méndiz Noguero, Ana María Sedeño, Ana Melendo, Ángel Luis Hueso Montón, Antonia del Rey Reguillo, Antonio Martínez Illán, Begoña Gutiérrez San Miguel, Bernardo Riego Amézaga, Carlos Maciá Barber, Cristina Manzano Espinosa, Daniel Sánchez Salas, Daniel Vela Valldecabres, Efrén Cuevas Álvarez, Elvira Loma, Enrique Salanova, Eva Gallardo, Fernando Luque, Francisco García Gómez, Francisco Segado Boj, Gonzalo M. Pavés, Javier Ortiz Echagüe, Joaquin Tomás Canovas Belchi, Jordi Alberich Pascual, Jorge Latorre Izquierdo, Jorge Marinho, Jorge Pedro Sousa, José Gabriel Ferreras Rodríguez, José Luis Campos García, Kepa Sojo Gil, Lorenzo Javier Torres Hortelano, Luis Castelo Sardina, Luis Del Tell Escolar, Luis Navarrete, Mar Marcos Molano, María A. Gabino Campos, María Concepción Mateos Martín, María Cristina Cañamero Alvarado, María Jesús Fernández Torres, María Pilar Yébenes Cortés, Mario de la Torre Espinosa, Mario Rajas Fernández, Marta Frago Pérez, Marta Redondo García, Miguel Ángel Huerta Floriano, Neftalí Pérez Franco, Patricio Pérez Rufi, Pedro Poyato, Pedro Sangro Colón, Pilar Irala Hortal, Rafael Gómez Alonso, Rafael Linares Palomar, Roberto Gelado Marcos, Ruth Gutiérrez, Silvia Herreros de Tejada Larrinaga, Sofía López Hernández, Valentín Serrano y Víctor Navarro Remesal.

Nos tocan vivir muchos retos, conformar una estructura de un monográfico al año (hemos publicado dos Los abrazos rotos de Pedro Almodóvar coordinado por Pedro Poyato y Narrativas de la crisis en cine coordinado por Antonio Sánchez-Escalonilla y Araceli Rodríguez, y el próximo será Miradas convergentes: la fotografía y sus interpretaciones en Humanidades y Ciencias Sociales coordinado por Javier Marzal Felici y Bernardo Riego Amézaga), mejorar la visibilidad y en definitiva que sea un encuentro en el que los autores y lectores se sientan tan satisfechos como nosotros. 